\title{
Reproducción de violencia de género en el ejercicio docente: análisis teórico de contenido orientado a la escuela básica chilena
}

\section{Reproduction of gender violence in the teaching profession: theoretical analysis of content oriented to the Chilean primary school}

\author{
*Javiera Cares Rojas., ${ }^{* *}$ Claudia Castro Morales., ${ }^{* * *}$ Paula Elizondo Rojas., \\ ****Karla González Cárdenas, Violeta Jara Quijada \& Felipe Jacob Marín Isamit ${ }^{1}$
}

Cares, J., Castro, C., Elizondo, P., González, K., Jara, V., \& Marín, F. (2021). Reproducción de violencia de género en el ejercicio docente: análisis teórico de contenido orientado a la escuela básica chilena. Revista Convergencia Educativa, (10-extra), diciembre, 34-50. http://doi.org/10.29035/rce.s10.34

[Recibido: 27 enero, 2021 / Aceptado: 23 octubre, 2021]

\section{RESUMEN}

El Seminario de Licenciatura, se basó en el supuesto que existen acciones ejecutadas por docentes de escuelas básicas que según un análisis teórico son potenciales reproductores de violencia de género. Se ha desarrollado una revisión teórica con el propósito central de analizar factores asociados a la reproducción de violencia de género, los cuales repercuten en acciones del profesorado en contexto de enseñanza básica. Centrados en el paradigma interpretativo con enfoque cualitativo, esta revisión teórica con análisis de contenido, se orienta desde el enfoque socio-crítico en etapa de discusión. Como principal conclusión surge la necesidad de promover una educación no discriminatoria en la enseñanza básica, en cuanto a roles y relaciones de género, en búsqueda de la igualdad, ideología y práctica no sexista, dando relevancia para lograr así el derecho a una educación de calidad con enfoque de género, donde el rol docente se posiciona en el centro de las responsabilidades.

Palabras clave: Enfoque de género, violencia de género, enseñanza básica, rol docente.

Este trabajo/artículo fue presentado en las jornadas Internacional de Investigación en Didáctica y Formación Inicial Docente JIIDFID UCM 2020.

Egresadas de la Escuela de Pedagogía en Educación General Básica del Departamento de Formación Inicial Escolar. Facultad de Ciencias de la Educación de la Universidad Católica del Maule. Talca-Chile.

* https://orcid.org/0000-0002-0487-2944 | javieracaresrojas@gmail.com

** https://orcid.org/0000-0001-7160-9014 | claucastromorales@gmail.com

*** https://orcid.org/0000-0003-0553-8566| paulaelizondo25@gmail.com

**** https://orcid.org/0000-0002-4350-5645 | karla.gocardenas@gmail.com

*****https://orcid.org/0000-0001-5745-7739|violeta.paz.jara.quijada@gmail.com

1 Investigador, director del seminario: Académico del Departamento de Formación Inicial Escolar. Facultad de Ciencias de la Educación de la Universidad Católica del Maule. Talca-Chile.

https://orcid.org/0000-0003-1314-7733 | fmarin@ucm.cl 
Cares, J., Castro, C., Elizondo, P., González, K., Jara, V., \& Marín, F. (2021). Reproducción de violencia de género en el ejercicio docente: análisis teórico de contenido orientado a la escuela básica chilena. Revista Convergencia Educativa, (10-extra), diciembre, 34-50. http://doi.org/10.29035/rce.s10.34

\section{ABSTRACT}

The Undergraduate seminar was based on the assumption that there are actions carried out by elementary school teachers who, according to a theoretical analysis, are potential reproducers of gender violence. A theoretical review has been developed with the central purpose of analyzing factors associated with the reproduction of gender violence, which have an impact on teacher actions in the context of primary education. Focused on the interpretive paradigm with a qualitative approach, this theoretical review with content analysis, is oriented from the socio-critical approach in the discussion stage. The main conclusion is the need to promote a non-discriminatory education in primary education, in terms of gender roles and relations, in search of equality, ideology and non-sexist practice, giving it the relevance to achieve the right to quality education with a gender perspective, where the teaching role is positioned at the center of responsibilities.

Key words: Gender Approach, Gender Violence, Basic Education, Teaching role.

\section{Introducción}

Para distintos autores y autoras, organizaciones internacionales y nacionales, la violencia de género es un problema que se encuentra presente en diversos aspectos dentro de nuestra sociedad, la cual afecta especialmente a las mujeres. La reproducción de esta violencia se puede ver reflejada, también, en la calidad y enseñanza de los establecimientos educacionales, impidiendo, ya sea implícita como explícitamente, la equidad de género.

Se debe tener en cuenta que, para dar lugar a estas situaciones de violencia de género, existe un sistema que sustenta y sostiene todo aquello. Desde los primeros años de vida de las personas, la escuela es el lugar de socialización más importante de niños y niñas, por lo cual, se debe tomar conciencia de cómo dentro del contexto escolar existen acciones y factores que reproducen dicha violencia.

Para el sustento de esta revisión bibliográfica se analizaron diversos estudios, los cuales permiten afirmar que toda agresión perpetrada contra una mujer tiene alguna característica que permite identificarla como violencia de género. Esto significa que está directamente vinculada a la desigual distribución del poder y a las relaciones asimétricas que se establecen entre varones y mujeres en nuestra sociedad, perpetuando la desvalorización de lo femenino y su subordinación a lo masculino (Rico, 1996, Alonso, et al. 2016).

\subsection{Justificación}

Desde la década de los 90', se comenzó a manifestar con fuerza un movimiento social que ha buscado visibilizar en Chile las demandas de las mujeres y también de las personas pertenecientes a la diversidad sexual (Barrientos et al., 2018).

Dicho fenómeno es definido por la Organización de las Naciones Unidas, como: 
Cares, J., Castro, C., Elizondo, P., González, K., Jara, V., \& Marín, F. (2021). Reproducción de violencia de género en el ejercicio docente: análisis teórico de contenido orientado a la escuela básica chilena. Revista Convergencia Educativa, (10-extra), diciembre, 34-50. http://doi.org/10.29035/rce.s10.34

Todo acto de violencia basado en la pertenencia al sexo femenino que tenga o pueda tener como resultado un daño o sufrimiento físico, sexual o psicológico para la mujer, así como las amenazas de tales actos, la coacción o la privación arbitraria de la libertad, tanto si se producen en la vida pública como en la vida privada (Organización de las Naciones Unidas [ONU], 1993, p. 2).

Según lo anterior, no se puede concebir la violencia de género como un problema aislado, pues repercute en todas las relaciones humanas, incluyendo los primeros años de educación formal que, en Chile, se enmarca en el rango de los 6 a 13 años de edad y se denomina Enseñanza Básica, similar a la Enseñanza Primaria definida en otros países. Los y las estudiantes, en esta etapa escolar, imitan comportamientos discriminatorios y violentos que observan en su entorno familiar y social, reproduciéndose como violencia directa o simbólica a nivel interpersonal en el espacio escolar (Trucco y Ullmann, 2015).

Es así como en Chile, gran parte de las violencias que ocurren a diario en los contextos escolares tienen que ver con descalificaciones, discriminaciones o agresiones dirigidas a estudiantes que no cumplen con un estereotipo de género.

Según organizaciones internacionales, esta "parece ser la forma de violencia más común en los centros educativos" (United Nations Educational, Scientific and Cultural Organization [UNESCO], 2018, Fundación Semilla, 2015, p. 11).

De esta forma, se torna relevante analizar la violencia de género como fenómeno presente en la enseñanza básica, con miras a proyectar orientaciones docentes visibilizando acciones y factores que durante siglos se han reproducido en la sociedad, y, por ende, también en las salas de clase. Tal como ha señalado Stanworth (1981; cit. en Flores, 2005):

La reproducción, que realizan los docentes, no opera de manera abierta, lo hace de forma invisible y eficiente. Se enseñan las mismas asignaturas tanto a niñas, como a niños, sin embargo, se da a entender que no necesitan adquirir el mismo dominio sobre ellas. A medida que se explica la materia, se dan ejemplos que privilegian a uno o a otro género, o bien se trabaja con textos cuyas ilustraciones hacen más referencia a un sexo que a otro (p.77).

Es, con referencia a lo mencionado, que la relevancia del tema a investigar radica en poder analizar cómo y por qué el objeto de estudio, el docente, continúa replicando acciones que se reflejan en la violencia de género vivida por las niñas y niños en Chile. Como estudiantes en formación, se comprende la relevancia de abordar esta temática desde diferentes dimensiones. Entre ellas, se destaca la formación inicial y continua del profesorado con miras al impacto que esta tiene en el rol docente y su práctica laboral. 
Cares, J., Castro, C., Elizondo, P., González, K., Jara, V., \& Marín, F. (2021). Reproducción de violencia de género en el ejercicio docente: análisis teórico de contenido orientado a la escuela básica chilena. Revista Convergencia Educativa, (10-extra), diciembre, 34-50. http://doi.org/10.29035/rce.s10.34

\section{Metodología}

Es a través del proceso de revisión teórica, utilizando una técnica de triangulación y análisis de contenido, que se logran levantar dos preguntas de investigación:

¿Cuáles son los factores asociados a la reproducción de la violencia de género que se discuten desde la investigación socioeducativa y desde las políticas públicas? y ¿Qué desafíos surgen desde las acciones que reproducen la violencia de género en el desempeño docente?

A partir de las preguntas de investigación, se estipula un objetivo general: analizar factores asociados a la reproducción de violencia de género, los cuales repercuten en acciones del profesorado en contextos de enseñanza básica. Para posteriormente levantar tres objetivos específicos: identificar factores asociados a la reproducción de la violencia de género en educación básica, categorizar las acciones asociadas a la reproducción de la violencia de género en educación básica a partir de las dimensiones de violencia $\mathrm{y}$, finalmente, discutir los desafíos a enfrentar por las y los docentes de enseñanza básica para contribuir en la erradicación de la violencia de género.

Para efectos de este seminario, se realizó un método investigativo en base a revisión bibliográfica a través del paradigma interpretativo, el cual hace profundizar y reflexionar sobre los diferentes motivos de los hechos. Se intenta comprender la realidad, considerando que el conocimiento no es neutral, sino que es relativo a los significados de los sujetos, esta interacción que se da tiene pleno sentido en la cultura y en la cotidianidad del fenómeno educativo (Pérez, 1994, cit. en Ricoy, 2006, p.17) y del paradigma socio-crítico, el cual, según Ricoy (2006):

Exigen del investigador una constante reflexión acción, implicando el compromiso del investigador/a desde la práctica para asumir el cambio y la liberación de las opresiones que generen la transformación social. Esto implica un proceso de participación y colaboración desde la autorreflexión crítica en la acción (p.17).

Por lo que, en primer lugar, se discutió y reflexionó sobre la labor docente en el aula y cómo esta influye en la formación de los estudiantes de educación básica. De esta manera se determinó el tema de investigación, el cual busca explicar cómo se relaciona el ejercicio docente con la reproducción de la violencia de género, identificando e interpretando a través de qué factores, puede estar siendo reproducida la violencia de género en las escuelas chilenas.

Para responder a lo anterior, se hizo una investigación con un enfoque cualitativo, considerando algunas características de este mismo, según López (2002), este tipo de investigación es de carácter holístico, puesto que abarca el fenómeno en su conjunto. Se analizó la problemática, donde se consideró desde los aspectos más generales a los particulares. El diseño de investigación fue emergente, es decir, se fue elaborando a 
Cares, J., Castro, C., Elizondo, P., González, K., Jara, V., \& Marín, F. (2021). Reproducción de violencia de género en el ejercicio docente: análisis teórico de contenido orientado a la escuela básica chilena. Revista Convergencia Educativa, (10-extra), diciembre, 34-50. http://doi.org/10.29035/rce.s10.34

medida que avanzaba la investigación, replanteando el problema, adaptando y ajustando este pertinentemente, así como también, se consideró el uso de categorías que se les atribuyó a cada uno de los elementos y/o dimensiones investigadas. Y desde luego, en este tipo de investigación "se realiza una revisión teórica, siendo la literatura útil para detectar conceptos claves y nutrirnos de ideas sobre métodos de recolección de datos y análisis, así como entender mejor los resultados, evaluar las categorías relevantes y profundizar en las interpretaciones" (Hernández et al., 2014. p.365).

\section{Resultados y discusión}

Dentro de los antecedentes teóricos y empíricos relacionados a la violencia de género, se destacan estudios realizados por la UNESCO (2018) y la Fundación Semilla (2015), las cuales declaran que existe bullying tanto para niños como niñas, sin embargo, este se manifiesta de diversas maneras para cada género, siendo más frecuente la violencia psicológica en niñas al ser ignoradas, excluidas, víctimas de rumores o propensas a sufrir ciberacoso, mientras que, los niños tienen mayor probabilidad de vivir violencia física. A esto, se suma la violencia sexual perpetuada por directores, maestros u otro estudiante.

La violencia de género se concibe como un problema de poder, es decir, nace de un sistema estructural de opresión de género, negando derechos básicos a mujeres y ubicándose en situación de exclusión social y desempoderamiento. Para Piotti (1989), resolver la discriminación a la mujer requiere de un proceso de transformación de la mentalidad colectiva que internaliza la ideología patriarcal, reformando así la idea que se tiene sobre los roles de varones y mujeres, siendo la educación, imprescindible para transformar estos roles. A raíz de esto, se considera relevante definir los conceptos asociados a este problema, tales como: patriarcado, feminismo, entre otros.

En cuanto al patriarcado, las investigadoras Ruiz \& Ruiz (2015), lo definen como sistema sociocultural que se basa en un conjunto de ideas, prejuicios, símbolos, costumbres e incluso leyes que considera a los varones como sujetos superiores a las mujeres, obteniendo así, el control en la familia, trabajo y aspectos sociales en general. En efecto de la vigencia del patriarcado, los varones interiorizan ser agresivos e imperativos, mientras que las mujeres asumen la mansedumbre y sumisión, siendo el patriarcado el marco normativo del machismo.

El machismo tiene raíces etimológicas en académicas feministas norteamericanas al evidenciar opresión de género en México, anunciando así que era un rasgo cultural único del varón de habla hispana. Este concepto se define como una etiqueta ante las conductas discriminatorias contra mujeres y otros varones que no poseen los requisitos de masculinidad hegemónica, defendiendo los privilegios de dominación masculina (Ruiz y Ruiz, 2015).

Respecto al feminismo, Salles (2002) menciona que este, ha colaborado a distintos campos de observación para las transformaciones culturales: la crítica a las normas que organizan la vida familiar, las 
Cares, J., Castro, C., Elizondo, P., González, K., Jara, V., \& Marín, F. (2021). Reproducción de violencia de género en el ejercicio docente: análisis teórico de contenido orientado a la escuela básica chilena. Revista Convergencia Educativa, (10-extra), diciembre, 34-50. http://doi.org/10.29035/rce.s10.34

reelaboraciones sobre lo privado y lo público y el cuestionamiento de los códigos morales, la violencia de género, entre otros.

Esta problemática se ve reflejada también en la escuela, entendiendo por violencia escolar un comportamiento impositivo, que tiene la intención de dominar y ejercer control sobre otro sujeto y que se da en un contexto interpersonal, pudiendo producir daño físico, psicológico o afectar el ámbito social (Costa \& Morales, 1998). De esta forma, se pueden reconocer distintos tipos de violencia escolar, entre ellos, la violencia de género.

Dentro de los factores asociados a la reproducción de la violencia de género en el aula, el Ministerio de la Mujer y Equidad de Género (s.f), señala que Chile, está suscrito a tratados de carácter internacional que buscan regular la situación de la violencia de género, entre ellas, La declaración sobre la eliminación contra la mujer, y El Comité de las Naciones Unidas para la eliminación de todas las formas de discriminación contra la mujer. Mientras que, a nivel educacional, Chile está suscrito a La Convención Contra las Discriminaciones en la Esfera de la Enseñanza, a los Objetivos de Desarrollo Sostenibles de la PNUD y la Convención Belém do Pará.

Existen, además, una serie de leyes y políticas públicas chilenas vigentes asociadas a la violencia de género, que establecen principios y obligaciones para los centros educativos que incluyen explícitamente el que la no discriminación, resguardo del derecho a educación de estudiantes embarazadas y madres, resguardando la educación integral en todos sus aspectos. Dentro de estas normativas se encuentran: Ley General de Educación ( $\left.\mathrm{N}^{\circ} 20.370\right)$, Ley de Inclusión Escolar ( $\left.\mathrm{N}^{\circ} 20.845\right)$ del 2015, Ley de Identidad de Género $\left(\mathrm{N}^{\circ} 21.120\right)$ del 2018, Ley contra la discriminación, más conocida como Ley Zamudio ( $\left.\mathrm{N}^{\circ} 20.609\right)$ del 2012, y, finalmente, la circular $N^{\circ} 0768$ en 2017. Pese a esto, Torres (2019) recalca que el sistema chileno tiende a invisibilizar las diferencias, especialmente a disidencias sexuales y/o personas pertenecientes al género binario, reprimiendo cualquier manifestación que no cumpla con el orden heteronormativo entorno escolar.

En la misma línea, en cuanto a las políticas ministeriales, las Bases Curriculares constituyen una parte importante del proceso de enseñanza aprendizaje, ya que, desde estas se formulan los llamados Objetivos de Aprendizaje (en adelante OA), permitiendo focalizar la acción en el aula (MINEDUC, 2012a). Al revisar los OA que hacen referencia a la violencia de género y su erradicación, se evidencia un solo objetivo correspondiente a la asignatura de Orientación, el cual se desarrolla desde tercero a sexto básico, sin modificación alguna, este dicta:

Manifestar actitudes de solidaridad y respeto, que favorezcan la convivencia, como: actuar en forma empática (poniéndose en el lugar del otro); utilizar un buen trato (por ejemplo, saludar, despedirse, pedir por favor); evitar y rechazar toda forma de violencia y discriminación ya sea por etnias, género, religión, nacionalidad, etc.; respetar el derecho de todos a expresar opiniones 
Cares, J., Castro, C., Elizondo, P., González, K., Jara, V., \& Marín, F. (2021). Reproducción de violencia de género en el ejercicio docente: análisis teórico de contenido orientado a la escuela básica chilena. Revista Convergencia Educativa, (10-extra), diciembre, 34-50. http://doi.org/10.29035/rce.s10.34

y pensar diferente; prestar ayuda especialmente a quien lo necesite; respetar el ambiente de aprendizaje (MINEDUC, 2012b. pp. 5-6).

Por su parte, los Objetivos de Aprendizaje Transversales (desde ahora OAT), establecen metas de carácter comprensivo y general para la educación escolar referidas al desarrollo personal, intelectual, moral y social de los estudiantes (MINEDUC, 2011c).

En cuanto a lo planteado anteriormente, Pilar Muñoz y Liliana Ramos, académicas de la Facultad de Educación de la Universidad Diego Portales, mencionan que, si bien Chile ha superado la desigualdad de acceso a la educación entre mujeres y hombres, siguen operando patrones que reproducen las representaciones tradicionales de género y de esa forma la desigualdad e inequidad de género. Esto se evidencia en las trayectorias educativas de las y los estudiantes, sus resultados, las opciones vocacionales marcadas por los estereotipos asociados a funciones femeninas y masculinas, la violencia de género, la discriminación y el embarazo adolescente, entre otros aspectos (Muñoz \& Ramos, 2018)

En cuanto a la Formación Inicial Docente, esta se ha transformado en un campo de estudio, y los procesos formativos en unidades de análisis, cuyos propósitos de corto, mediano y largo alcance visibilizan el éxito o fracaso de las estrategias político-educativas relacionadas al enfoque de género (Lizana, 2009), así (respecto a la incorporación del enfoque de género en estas) la ausencia de estándares obligatorios de calidad ha contribuido a mantener la reproducción de las ideologías de género en la educación como un tema pendiente.

En materia de formación y capacitación docente, conforme al MINEDUC, se realiza continuamente una formación en enfoque de género para docentes y la comunidad educativa, a través de cursos sobre discriminación, escuela inclusiva, enfoque de género, sexualidad y diversidad sexual en el aula para docentes a nivel nacional, entregados en las plataformas del Centro de Perfeccionamiento, Experimentación e Investigaciones Pedagógicas (en adelante CPEIP) (MINEDUC, s.f.). Desde el CPEIP declaran comprometerse con promover la igualdad de trato y de oportunidades a través de todas sus acciones formativas dirigidas a docentes y a directivos, así como también, a través de iniciativas orientadas a la formación de futuros profesores (Centro de Perfeccionamiento, Experimentación e Investigaciones Pedagógicas [CPEIP], s.f.). Aun así, en consideración de lo anterior, se hace plenamente vigente considerar la recomendación de la UNESCO en su visión y acción para la educación superior del siglo XXI:

Se requieren más esfuerzos para eliminar todos los estereotipos fundados en el género en la educación superior, tener en cuenta el punto de vista del género en las distintas disciplinas, consolidar la participación cualitativa de las mujeres en todos los niveles y las disciplinas en que están insuficientemente representadas, e incrementar sobre todo su participación activa en la adopción de decisiones (UNESCO, 1998). 
Cares, J., Castro, C., Elizondo, P., González, K., Jara, V., \& Marín, F. (2021). Reproducción de violencia de género en el ejercicio docente: análisis teórico de contenido orientado a la escuela básica chilena. Revista Convergencia Educativa, (10-extra), diciembre, 34-50. http://doi.org/10.29035/rce.s10.34

Respecto a los factores que reproducen la violencia de género en el contexto escolar, se han revisado: Proyecto Educativo Institucional (en adelante PEI) dado su importancia en la orientación del quehacer escolar y, en otro punto, las asignaturas y textos escolares, pues se ha documentado que estos acentúan y, por lo tanto, perpetúan estereotipos de género.

Luego, se analizaron cómo aquellas características desde el plano personal del docente se vuelven factores que reproducen la violencia de género. Estos son, el currículum oculto y currículum oculto de género (en adelante COG).

Se entiende como currículum oculto como aquello que "hace referencia a las normas y valores enseñados en la escuela de manera implícita, sin que sean parte de los fines, objetivos o contenidos explícitamente planteados por el profesorado" (Guerrero et al., 2011, p. 49). Por su parte, el currículum oculto de género se define como el "conjunto interiorizado y no visible, oculto para el nivel consciente, de construcciones de pensamiento, valoraciones, significados y creencias que estructuran, construyen y determinan las relaciones y las prácticas sociales de y entre hombres y mujeres" (Lovering \& Sierra, 2009, párr. 15). Este se caracterizará, bajo la visión de varias autoras (Estrada et al., 2004, Guerrero et al., 2011, Lovering \& Sierra, 2009, Violi, 1991), en un desglose de cuatro ejes esenciales desde los cuales se permite comprender la labor docente como un factor reproductor de la violencia de género, estos son: esencialismo, androcentrismo, roles y formatos de la participación y uso del lenguaje.

\subsection{Discusión}

Dado lo anterior, el equipo investigativo desarrolló discusiones a modo de análisis crítico de los resultados asociados a cada dimensión y sus categorías: políticas educativas, comunidad educativa y rol docente. Para efectos de las discusiones, la FID se considerará dentro de la dimensión Rol Docente.

- Leyes y políticas públicas.

En esta dimensión se destacan las semejanzas expuestas por el MINEDUC, acuerdos internacionales y leyes establecidas en nuestro país, las cuales buscan garantizar una educación de calidad, inclusiva y equitativa (Mineduc, 2017).

Pese a esto, Vivar (2017) declara que aún no se logran cambios significativos en las políticas públicas chilenas:

Las políticas públicas chilenas han planteado y trabajado por la equidad de género desde la perspectiva cuantitativa, relacionada con el acceso a la educación de mujeres (escolaridad, alfabetización, matrícula, egreso, aprobación, etc.); pero no han alcanzado cambios significativos en lo que corresponde a la socialización de género, traducido en la reproducción de estereotipos 
Cares, J., Castro, C., Elizondo, P., González, K., Jara, V., \& Marín, F. (2021). Reproducción de violencia de género en el ejercicio docente: análisis teórico de contenido orientado a la escuela básica chilena. Revista Convergencia Educativa, (10-extra), diciembre, 34-50. http://doi.org/10.29035/rce.s10.34

y pautas de comportamiento respecto de lo que se ha considerado "tradicional" en atributos, papeles y jerarquía social de hombres y mujeres (p. 37).

Se puede considerar, entonces, con lo expuesto en esta revisión teórica con análisis de contenidos, que las leyes y políticas públicas respecto a la violencia de género no logran atender las necesidades actuales, pues no se enfrenta el problema general de este fenómeno. Existe un vacío normativo en la tipificación de los distintos tipos de violencia de género en Chile. Esta insuficiencia ha dejado sin normativa ni sanción a la violencia obstétrica, la violencia sobre mujeres migrantes, la violencia económica, la violencia psicológica y el acoso sexual en contextos educativos, por lo tanto, la normativa vigente no resguarda los derechos esenciales para vivir y estudiar en un espacio libre de violencia de género (Lamadrid et al., 2019).

- Comunidad educativa.

La reproducción de la cultura dominante a través de las acciones pedagógicas rutiniza la cultura escolar, codificando, homogeneizando y sistematizando el mensaje por medio de quienes lo transmiten (Bourdieu \& Passeron, 1975).

La circular llamada "Derechos de niñas, niños y estudiantes trans en el ámbito de la educación del MINEDUC (2017), asevera que:

Asegurar el derecho a la educación de niños, niñas y estudiantes, tanto en el acceso como en la trayectoria educativa, significa atender sus necesidades y diversidades personales y colectivas, creando espacios seguros y respetuosos de su dignidad que favorezcan el desarrollo integral ( $p$. 1).

Frente a esto, la agrupación Nuestra Clase (2017) discrepa del MINEDUC y cuestiona a través de la siguiente declaración:

¿Cómo asegurar el derecho a la educación, respetando la dignidad de los jóvenes, o niños con identidades y sexualidades divergentes, si en la sociedad de conjunto, está arraigadísimo el machismo y la heteronormatividad patriarcal? ¿Cómo asegurar este derecho, si las concepciones de las iglesias, de la mano del capitalismo y el Estado, siguen teniendo peso en las políticas educativas? (en línea).

De acuerdo a lo expuesto anteriormente, podemos entender que la escuela funciona como un escenario que posiciona y reproduce el patriarcado como orden social, bajo el cual el género masculino domina al femenino e incluso se presenta como el punto de referencia (Echeverría, 2017). 
Cares, J., Castro, C., Elizondo, P., González, K., Jara, V., \& Marín, F. (2021). Reproducción de violencia de género en el ejercicio docente: análisis teórico de contenido orientado a la escuela básica chilena. Revista Convergencia Educativa, (10-extra), diciembre, 34-50. http://doi.org/10.29035/rce.s10.34

- Rol docente

En relación a la labor docente, al hablar sobre FID, se pretende resaltar las consecuencias en la formación de niños y niñas, cuando los docentes no son conscientes de su capacidad de reproducir valores, actitudes, conductas y habilidades sociales sin la crítica necesaria para que la educación sea verdaderamente un apoyo para la transformación personal y social (Lovering \& Sierra, 2009).

Pese a estos esfuerzos, hay ciertos factores que no se pueden estandarizar ni evaluar, por ejemplo, el COG y la conducta y respuesta emocional de parte de los docentes. El primero resulta ser transversal en la reproducción de la violencia de género, ya que está íntimamente relacionada con la educación formal y no formal, instaurándose en ellas (Lovering \& Sierra, 2009). Mientras que, en el segundo caso, la investigación internacional demuestra que las expectativas de aprendizaje que poseen los y las docentes sobre los niños y niñas, influyen directamente en las actitudes de los estudiantes, un ejemplo de aquello es la justificación de agresión física en el caso de los hombres y la expectativa de sumisión en mujeres (Cortés, 2018).

La Agencia de Calidad de la Educación (en adelante ACE) señala que desde los 5 años de edad niños y niñas creen que estas últimas tienen más probabilidad de no gustarles las matemáticas, provocando un bajo rendimiento en el área. Además, se agrega el problema declarado por diversas investigaciones, donde señalan que para los hombres la lectura se considera una actividad feminizada, en especial los textos de ficción (Agencia de Calidad de la Educación [ACE], 2018).

A pesar de que los resultados SIMCE muestran un avance significativo en la eliminación de la brecha de género, el secretario general de la ACE declara que el problema se cimenta bajo aspectos derechamente culturales:

Dado que hombres y mujeres tienen capacidades biológicas similares para enfrentar las distintas áreas del aprendizaje, la raíz de estas diferencias radica en elementos culturales tan enraizados que muchas veces no tenemos conciencia de ellos. Así, uno de los resultados más importantes de nuestro estudio de buenas prácticas para la reducción de la brecha de género es justamente la poca visibilidad del problema en los establecimientos educacionales (Henríquez, 2018).

\section{Conclusiones}

Para responder a la primera pregunta de investigación que hace referencia a cuáles son los factores asociados a la reproducción de la violencia de género que se discuten desde la investigación socioeducativa y desde las políticas públicas, se comprendieron diversos factores relacionados a la reproducción de la violencia de género en la escuela. Se determinó que estos factores pueden desprenderse de distintas categorías de análisis. Estas, como ya han sido planteadas previamente, son las siguientes: acuerdos internacionales, leyes y políticas públicas, bases curriculares, PEI, tipo de dependencia, aspectos 
Cares, J., Castro, C., Elizondo, P., González, K., Jara, V., \& Marín, F. (2021). Reproducción de violencia de género en el ejercicio docente: análisis teórico de contenido orientado a la escuela básica chilena. Revista Convergencia Educativa, (10-extra), diciembre, 34-50. http://doi.org/10.29035/rce.s10.34

socioculturales, asignaturas y textos escolares, la formación inicial docente, el currículum oculto, la conducta y la respuesta emocional.

La principal conclusión que se desprende de la presente investigación radica en comprender que el docente no es el único sujeto responsable de la erradicación de la violencia de género en el aula, sino que también las categorías, de carga política, social y cultural consideradas en esta investigación, funcionan como factores que inciden en este problema.

De la primera dimensión estudiada, políticas públicas, se concluye que, a pesar de que Chile esté suscrito a diversos acuerdos internacionales, y posea legislación que apunte a sancionar la violencia contra la mujer, estas no logran un impacto significativo en la sociedad, pues la justicia chilena sanciona de forma retroactiva y no ataca este problema de raíz. Esto influye directamente en la educación, puesto que crea una realidad donde la discriminación, inequidad y justicia social perpetúan la reproducción de la violencia de género, internalizando esta en niños y niñas, restringiendo así también su derecho de educarse en un ambiente seguro, libre de violencia, respetuoso y con equidad.

Se concluye, además, que los únicos avances desde el Mineduc abordando esta temática solo son propuestas y sugerencias, y no acciones concretas a desarrollar en aula; sumado a esto, los márgenes de libertad de enseñanza para los establecimientos hacen que estos transmitan ideologías y valores que quedan a criterio de cada equipo directivo, siendo estas ideologías las que implícitamente contribuyen a la reproducción de la violencia de género.

En materia de Formación Inicial Docente, se concluye que existe una carencia o una deuda con el enfoque de género, pues no se encuentran estándares básicos que apunten a una erradicación de la violencia de género. Sin embargo, se destacan positivamente los intereses desde la CNA por incluir estándares de género desde el año 2020 para las futuras acreditaciones de carreras de pedagogía, en respuesta a los nuevos proyectos provenientes de MinMujeryEG y Mineduc.

Se concluye que el ejercicio docente es uno de los factores más urgentes a atender para contribuir a la erradicación de la violencia de género, pues es éste quien debe asegurar con sus acciones y omisiones un ambiente libre de sexismo. El currículum oculto de género es el factor que más incide en la reproducción de este tipo de violencia, entendiendo esta como una violencia sistémica contra las mujeres. La repercusión que conlleva esta violencia en los estudiantes es de suma importancia, porque define las transformaciones de sentir, pensar y actuar en la construcción de su personalidad y en sus relaciones sociales.

Finalmente, se concluye que el Estado no demuestra mayor interés al no fiscalizar ni velar por el cumplimiento de los principios educativos que sustenta la erradicación de la violencia de género, dejando en obligatoriedad muchos aspectos sexistas al momento de la enseñanza. Así, la práctica docente está respaldada por la sociedad en la cual se desarrolla, y cuenta, además, con una formación inicial y continua 
Cares, J., Castro, C., Elizondo, P., González, K., Jara, V., \& Marín, F. (2021). Reproducción de violencia de género en el ejercicio docente: análisis teórico de contenido orientado a la escuela básica chilena. Revista Convergencia Educativa, (10-extra), diciembre, 34-50. http://doi.org/10.29035/rce.s10.34

del profesorado que no apuntan a combatir la violencia hacia la mujer con medidas claras y concretas en el aula.

Los desafíos surgen desde las acciones que reproducen la violencia de género en el desempeño docente. Estos desafíos se presentarán de acuerdo con las dimensiones analizadas anteriormente. Desde la dimensión de políticas educativas se desprende la necesidad de considerar a profesores y profesoras en la toma de decisiones, otorgándoles un rol importante, vinculante y decisivo en la construcción de políticas educativas para el país. El Mineduc debe ser una entidad fiscalizadora y reguladora, que asegure que todos los establecimientos de Chile puedan tener una educación equitativa sin sesgos de género y no sexista.

En la dimensión correspondiente a la comunidad educativa los desafíos surgen desde la necesidad de poner en práctica instrumentos que aborden las brechas de género y violencia hacia la mujer de manera integral teniendo en consideración, la prevención, educación, investigación y sanción tanto al interior, como fuera de los espacios educativos. Dicho lo anterior se plantean los siguientes desafíos: la elaboración de los PEI debe apuntar a lograr una equidad de género y no sexista, esto debe verse reflejado tanto en el reglamento interno, como en los protocolos de convivencia escolar. Realizar test psicológicos obligatorios al equipo docente y a asistentes de la educación, para que se puedan detectar y predecir, de manera temprana, estilos conductuales inapropiados (machistas, sociópatas y/o pedófilos).

Desde la dimensión del rol docente, nace el desafío de establecer criterios de calidad explícitos en relación con la formación con equidad de género, orientados a la creación de cátedras específicas, contenidos transversales y prácticas educativas libres de estereotipos en la malla curricular de cada carrera. Además, emerge la necesidad de entregar formación de género en actividades académicas estructuradas y explícitas en los currículos de FID por las diferentes carreras de pedagogía en Chile.

Finalmente, en relación con las labores ejecutadas en el aula, se desprende el desafío de diversificar el tipo de actividades desarrolladas en esta, privilegiando aquellas que favorezcan la reflexión y el pensamiento crítico por sobre la repetición de contenidos que puedan reproducir la violencia de género. Así mismo, los docentes deben ser capaces de adecuar los recursos e identificar los sesgos de género en el currículum explícito, pudiendo modificar su enseñanza a favor de una educación no sexista.

Como mujeres, a lo largo de nuestra vida, hemos podido evidenciar la violencia hacia nosotras, la mayoría de las veces de forma implícita, vivenciada en distintos planos de nuestro desarrollo, del cual no queda exenta nuestra etapa escolar. Tras realizar esta investigación, surgen emociones de rabia, tristeza e impotencia al comprender la inoperancia del Estado y sus leyes muertas, las cuales demuestran que la mujer sigue en estado de indefensión e inferioridad ante la violencia machista. Nos comprendemos violentadas, una y otra vez, por el organismo que debería resguardar nuestra integridad. Así, percibimos, como personas, 
Cares, J., Castro, C., Elizondo, P., González, K., Jara, V., \& Marín, F. (2021). Reproducción de violencia de género en el ejercicio docente: análisis teórico de contenido orientado a la escuela básica chilena. Revista Convergencia Educativa, (10-extra), diciembre, 34-50. http://doi.org/10.29035/rce.s10.34

la necesidad de poner sobre la mesa esta problemática en los diversos ámbitos de la sociedad y, como futuras docentes, instaurar una educación con equidad de género.

Como profesoras, creemos fuertemente que la lucha contra la violencia de género desde las escuelas debe ser, primeramente, contra el patriarcado, sin olvidar que es el capitalismo el que permite la existencia de esta forma de dominación y denostación hacia nosotras. Luchar contra el acoso y la violencia hacia nuestras vidas y cuerpos es luchar contra la educación de mercado sexista que nos educa para obedecer, aceptar la violencia y la desigualdad. Si el mercado educativo necesita del patriarcado, la nueva educación pública necesita del feminismo.

\section{REFERENCIAS BIBLIOGRÁFICAS}

Agencia de Calidad de la Educación. (2018). Estudio: Buenas prácticas en la reducción de las brechas de género en resultados Simce de Comprensión de Lectura y Matemática $I^{\circ}$ medio. http://archivos.agenciaeducacion.cl/Estudio_Brechas_de_Genero.pdf

Alonso, C., Cacho, R., González, I., Herrera., \& Ramírez, J. (2016) Guía de buen trato y prevención de la violencia de género: protocolo de actuación en el ámbito educativo. Junta de Andalucía. Consejería de educación. Dirección General de Participación y Equidad. https://www.observatoriodelainfancia.es/oia/esp/documentos_ficha.aspx?id=5027

Barrientos, P. Andrade, D., \& Montenegro, C (2018). La formación docente en género y diversidad sexual: tareas pendientes. Cuaderno de Educación, (81), 1-13. https://www.researchgate.net/profile/Pablo-BarrientosSaavedra/publication/341161632_La_formacion_docente_en_genero_y_diversidad_sexual_Tareas _pendientes/links/5eb18d30299bf18b9595f5a2/La-formacion-docente-en-genero-y-diversidadsexual-Tareas-pendientes.pdf

Bourdieu, P., \& Passeron, J.C. (1995). La Reproducción. Elementos para una teoría del Sistema de Enseñanza. Distribuciones Fontamara, S.A. https://socioeducacion.files.wordpress.com/2011/05/bourdieupierre-la-reproduccion1.pdf

CPEIP. (s.f.). CPEIP se compromete con la equidad de género. Chile: Centro de Perfeccionamiento, Experimentación e Investigaciones Pedagógicas. https://www.cpeip.cl/equidad-de-genero/

Cortés, I (2018). Guía para la no discriminación en el contexto escolar. Superintendencia de Educación de Chile. https://bibliotecadigital.mineduc.cl/handle/20.500.12365/433 
Cares, J., Castro, C., Elizondo, P., González, K., Jara, V., \& Marín, F. (2021). Reproducción de violencia de género en el ejercicio docente: análisis teórico de contenido orientado a la escuela básica chilena. Revista Convergencia Educativa, (10-extra), diciembre, 34-50. http://doi.org/10.29035/rce.s10.34

Costa, M., \& Morales, J. (1998). ¿Por qué hay niños que cuando jóvenes llegan a comportarse violentamente? Claves para comprender el desarrollo de la violencia. Anuario de Psicología Jurídica. 163-179. https://journals.copmadrid.org/apj/archivos/43985.pdf

Echeverría, S (2017). Sexismo y estereotipos de género en educación: el caso del 'primer foco de luz de la nación' y el predominio de su cultura escolar. Estudio de caso sobre la cultura escolar del Instituto Nacional y su dimensión de género. [Tesis de pregrado]. Universidad de Chile. https://repositorio.uchile.cl/handle/2250/152443

Estrada, C., Serón, E., \& Yzerbyt, V. (2004). Efecto del esencialismo psicológico sobre las teorías ingenuas de las diferencias grupales. Psicothema, 16(2), 181-186. http://www.psicothema.es/pdf/1180.pdf

Flores, R. (2005). Violencia de Género en la Escuela: sus efectos en la identidad, en la autoestima y en el proyecto de vida. Revista Iberoamericana de Educación, (38), 67-86. https://rieoei.org/historico/documentos/rie38a04.pdf

Fundación Semilla. (2015). Violencias de género, otra mirada a la brecha escolar. Fundación Semilla. https://fundacionsemilla.cl/wp-content/uploads/2015/12/Violencias-de-G\%C3\%A9nero-OtraMirada-a-la-Brecha-Escolar.pdf

Guerrero, E., Hurtado, V., Azua., \& Provoste, P. (2011). Material de Apoyo con Perspectiva de Género para Formadores y Formadoras. Centro de Perfeccionamiento, Experimentación e Investigaciones Pedagógicas del Ministerio de Educación. Hexagrama consultoras. http://www.brunner.cl/wpcontent/uploads/2013/11/curriculo-de-genero-oculto-para-profesoras-es.pdf

Henríquez, C. (2018). La Educación y la Brecha de Género. El Mostrador. https://www.elmostrador.cl/noticias/opinion/2018/03/09/la-educacion-y-la-brecha-degenero/

Hernández, R., Fernández, C., \& Baptista,. M. (2014). Metodología de la investigación (6ta ed.) Mc Graw Hill Education.

Lamadrid, S., Retamal, P., \& Zagal, G. (2019). Revolución feminista en Educación no sexista. Aun creemos en los sueños.

Lizana, V. (2009). Una relación invisibilizada en los contextos de formación docente inicial: La identidad profesional desde una perspectiva de género. REICE. Revista Iberoamericana sobre Calidad, Eficacia y Cambio en Educación, 7(3),69-81. https://revistas.uam.es/index.php/reice/article/view/5402

López, F. (2002). El análisis de contenido como método de investigación. XXI, Revista de Educación, 4, 167180. http://rabida.uhu.es/dspace/bitstream/handle/10272/1912/b15150434.pdf?sequence1 
Cares, J., Castro, C., Elizondo, P., González, K., Jara, V., \& Marín, F. (2021). Reproducción de violencia de género en el ejercicio docente: análisis teórico de contenido orientado a la escuela básica chilena. Revista Convergencia Educativa, (10-extra), diciembre, 34-50. http://doi.org/10.29035/rce.s10.34

Lovering, A., \& Sierra, G (2009). El currículum oculto de género. Educar. Revista de educación, (7), 1-7. https://www.igualdadycalidadcba.gov.ar/SIPEC-

CBA/PolSocioeducativas/Documentos/ESI/BibliotecaDigital/8-

El\%20curriculum\%20oculto\%20de\%20genero.pdf

Ministerio de Educación. (s.f.). Educación para la Igualdad de Género. Plan 2015 - 2018. Ministerio de Educación: Unidad de Equidad de Género. https://www.mineduc.cl/wpcontent/uploads/sites/19/2017/01/CartillaUEG.pdf

Ministerio de Educación. (2011c) Objetivos Transversales. https://viancep2012.files.wordpress.com/2012/01/objetivos-transversales-bases-curriculares2012.pdf

Ministerio de Educación. (2012a). Bases curriculares. https://www.curriculumnacional.cl/portal/Documentos-Curriculares/Bases-curriculares/

Ministerio de Educación. (2012b). Progresión de objetivos de aprendizaje de Orientación de $1^{\circ}$ a $6^{\circ}$ básico. https://www.curriculumnacional.cl/portal/Documentos-Curriculares/Progresiones-deaprendizaje/71260:Progresion-de-objetivos-de-aprendizaje-para-Orientacion-de-1-a-6-basico

Ministerio de Educación. (2017). Cartilla Enfoque de Género: Incorporación de los instrumentos de Gestión Escolar. https://www.curriculumnacional.cl/portal/Secciones/Biblioteca-EscolarUCE/228065:Enfoque-de-genero-incorporacion-en-los-instrumentos-de-Gestion-Escolar

Muñoz, P., \& Ramos, L. (2018). Informe anual sobre Derechos Humanos en Chile 2018. Universidad Diego Portales. https://derechoshumanos.udp.cl/informe-anual/informe-anual-sobre-derechoshumanos-en-chile-2018/

Nuestra Clase. (2017). ¿Educación machista de la iglesia o educación no sexista de las profesoras? La Izquierda Diario. https://www.laizquierdadiario.cl/Educacion-machista-de-la-iglesia-oEducacion-no-sexista-de-las-profesoras.

Organización de las Naciones Unidas. (1993). Declaración sobre la eliminación de la violencia contra la mujer. https://www.ohchr.org/sp/professionalinterest/pages/violenceagainstwomen.aspx

Piotti, D. (1989). La ideología patriarcal: El rol de la educación. Sociológica, 4(10), 1-10. http://www.sociologicamexico.azc.uam.mx/index.php/Sociologica/article/view/986/958

Rico, N. (1996). Violencia de Género: un problema de Derechos Humanos. CEPAL https://repositorio.cepal.org/bitstream/handle/11362/5855/S9600674_es.pdf 
Cares, J., Castro, C., Elizondo, P., González, K., Jara, V., \& Marín, F. (2021). Reproducción de violencia de género en el ejercicio docente: análisis teórico de contenido orientado a la escuela básica chilena. Revista Convergencia Educativa, (10-extra), diciembre, 34-50. http://doi.org/10.29035/rce.s10.34

Ricoy, C. (2006). Contribución sobre los paradigmas de investigación. Educação. Revista do Centro de Educação, 31(1), 11-22. https://www.redalyc.org/pdf/1171/117117257002.pdf

Ruiz, S. y Ruiz, S (2015). Machismo misoginia patriarcado una reflexión desde la terapia narrativa. Revista $\begin{array}{lllll}\text { electrónica: } \quad \text { Procesos } & \text { Psicológicos } \quad y \quad \text { Sociales, } & 11(1), & 1-31 .\end{array}$ https://www.uv.mx/psicologia/files/2015/09/Sara-E.-Ruiz-Vallejo-Susana-Ruiz-Pimentel.pdf

Salles, V. (2002). Estudios sobre las mujeres y las relaciones de género en México: Aportes desde diversas disciplinas. El Colegio de México. https://doi.org/10.2307/j.ctvhn0b7g

Silva-Peña, I. (2010). Repensando la escuela desde la coeducación. Una mirada desde Chile. Revista Venezolana de Estudios de la Mujer., 15(34), 161-176. http://ve.scielo.org/scielo.php?script=sci_arttext\&pid=S1316-37012010000200009

Trucco, D., \& Ullmann H. (2015). Juventud: realidades y retos para un desarrollo con igualdad. CEPAL. http://www.cepal.org/es/publicaciones/juventud-realidades-retos-un-desarrollo-igualdad

United Nations Educational, Scientific and Cultural Organization. (1998). La educación superior en el siglo XXI: Visión y acción. https://unesdoc.unesco.org/ark:/48223/pf0000113878_spa

United Nations Educational, Scientific and Cultural Organization. (2018). School violence and bullying: Global status and trends, drivers and consequences. https://www.observatoriodelainfancia.es/ficherosoia/documentos/5805_d_BULLYING_UNESCO. pdf

Violi, P. (1991). El infinito singular. Cátedra $\quad$ S.A. https://www.mujerpalabra.net/libros/pdf/PatriziaVioli_Elinfinitosingular.pdf

Vivar, H. (2017). Violencia en la escuela: Significados otorgados por profesores y profesoras a la violencia de género al interior de los establecimientos educacionales [Tesis de magíster]. Universidad de Chile, Santiago, Chile. 


\section{Datos de correspondencia}

Felipe Jacob Marín Isamit

Magíster en Educación de las Ciencias Mención Química

Universidad Católica del Maule. Talca-Chile.

Dirección postal: 3660000

ORCID: https://orcid.org/0000-0003-1314-7733

Email: fmarin@ucm.cl 